\title{
Correction to: Ultrasound-guided transversus abdominis plane block (US-TAPb) for robot-assisted radical prostatectomy: a novel '4-point' technique-results of a prospective, randomized study
}

\author{
Fabrizio Dal Moro ${ }^{1} \cdot$ Luca Aiello $^{2} \cdot$ Paola Pavarin ${ }^{2} \cdot$ Fabio Zattoni $^{1}$
}

Published online: 14 August 2018

๑) Springer-Verlag London Ltd., part of Springer Nature 2018

\section{Correction to: Journal of Robotic Surgery https://doi.org/10.1007/s11701-018-0858-6}

The author would like to correct the errors in the publication of the original article due to multiple small errors in scientific content of article due to author oversight. Errors do not change or invalidate the conclusion of the article.

The corrected details are given below for your reading.

In Abstract, “... without (group A, 50 patients) or with US-TAPb (group B, 50 patients)..." should be changed to “... with (group A, 50 patients) or without US-TAPb (group B, 50 patients)...”.

The following sentence "Seven patients, all in group A, received $100 \mathrm{mg}$ of Tramadol. In group B, only one patient received $100 \mathrm{mg}$ Tramadol (first and second post-operative days) due to surgical complications" should be deleted from abstracts, so abstracts should be:

\begin{abstract}
Several works stress the importance of ultrasound-guided transversus abdominis plane block (TAPb) for post-operative analgesia and its versatility in all types of abdominal surgery, thanks to laparotomy and laparoscopy. The aim of this study was to evaluate the impact of $\mathrm{TAPb}$ on intra- and post-operative analgesia in the first $24 \mathrm{~h}$ after robot-assisted radical prostatectomy (RARP). TAPb is a new local anesthetic technique which provides analgesia after abdominal
\end{abstract}

The original article can be found online at https://doi.org/10.1007/ s11701-018-0858-6.

Fabrizio Dal Moro

fabrizio.dalmoro@unipd.it

1 Department of Surgery, Oncology

and Gastroenterology-Urology, University of Padova, Via

Giustiniani 2, 35128 Padova, Italy

2 Department of Anesthesiology, Azienda Ospedaliera di Padova, Padova, Italy surgery. It involves injection of local anesthetic into the plane between the transversus abdominis and the internal oblique muscles. TAPb can be performed according to a landmark technique, either through the lumbar triangle or with ultrasound guidance. We evaluated the intra- and post-operative analgesic efficacy of TAPb in 100 ASA I-III patients undergoing RARP under general anesthesia with (group A, 50 patients) or without US-TAPb (group B, 50 patients), in the first 24 post-operative hours. After induction of general anesthesia, US-TAPb was performed in 50 selected patients. All patients received post-operative analgesia (Paracetamol $1 \mathrm{~g}$ ) three times a day. Tramadol and Ketoprofen were used as rescue drugs if the Numerical Rating Scale test was $>3$. No complications were recorded during block performance. A significant reduction was seen in the need to administer intraoperative opioids, and in the occurrence of post-operative pain or post-operative drug consumption in patients receiving US-TAPb. In conclusion, US-TAPb provided highly effective intra- and post-operative analgesia in the first $24 \mathrm{~h}$ after RARP. A further prospective study is necessary to assess the best protocol for all patients.

In Materials and methods, Subtitle "Technique (group B)" should be changed to "Technique (group A)".

In Fig. 2, "Analysed $(\mathrm{n}=0)$ " should be changed to "Analysed ( $\mathrm{n}=50)$ " in both groups. The revised figure (Fig. 2) is placed in the following page. 


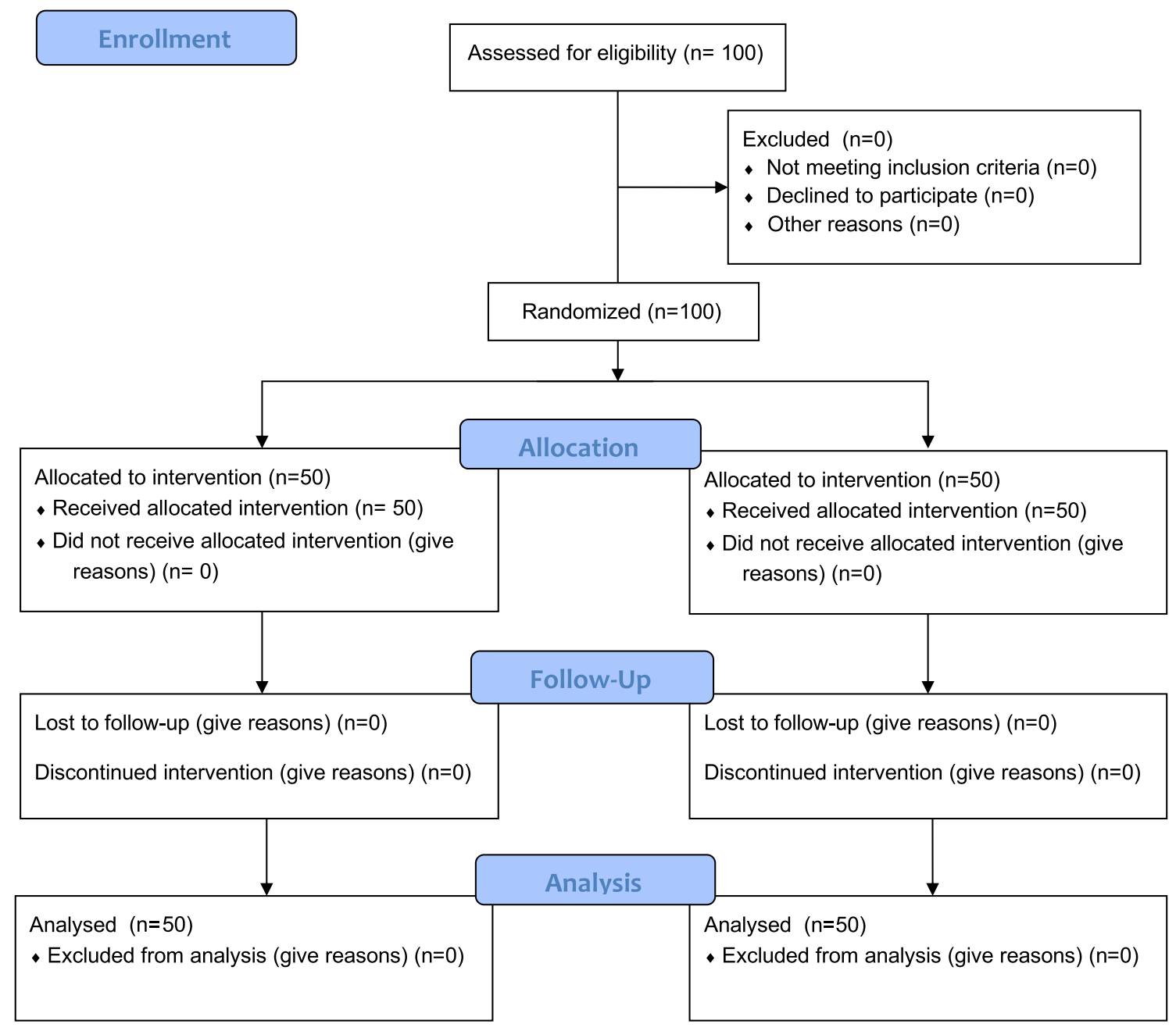

Fig. 2 CONSORT flow diagram 